\title{
II. Die Entwicklung des Schuldenstaats
}

\section{Staatsbankrotte in Geschichte und Gegenwart}

Am Anfang war der Staatsbankrott. Als Napoleon am 27. Oktober 1806 in Berlin einzog, blieben die Verbindlichkeiten der preußischen Krone unbeglichen ${ }^{1}$. Der Staatsbankrott Preußens von 1806 war weder der einzige noch der größte und schon gar nicht der letzte in der deutschen Geschichte. Allein in der ersten Hälfte des 20. Jahrhunderts war der deutsche Nationalstaat zweimal - als Folge der Finanzierung der beiden Weltkriege - insolvent ${ }^{2}$.

Staatsbankrotte sind in der Geschichte alles andere als selten. Den Rekord hält Spanien. Als sich das spanische Weltreich noch auf dem Höhepunkt seiner Macht befand, stellte Philipp II. gleich dreimal (1557, 1575 und 1596) die Zinszahlungen ein. Allein im 19. Jahrhundert wiederholte Spanien diese Praxis sechsmal. Auch Frankreichs Weg zur Weltmacht war bis 1789 mit acht Insolvenzen gepflastert. Nach dem Sturm auf die Bastille lehnten die französischen Revolutionäre den Schuldendienst für das alte Regime ebenso ab wie die russischen Revolutionäre 1917. Vermutlich war der russische Staatsbankrott von 1917/18 der größte der Weltgeschichte.

Eine Reihe von Ländern zeigt eine erstaunliche Kontinuität im ruinösen Umgang mit ihren Finanzen. Als Argentinien 2001/02 seine Zahlungen für den Kapitaldienst einstellte, blickte es auf eine lange Tradition vergleichbarer Vorgänge zurück. Ähnlich verhält es sich mit Griechenland: Die griechischen Bankrotte lassen sich seit der Unabhängigkeit des Landes im Jahr 1821 kaum noch zählen.

Die historischen und aktuellen Beispiele verdeutlichen, dass der Staatsbankrott in der Regel einer hohen Verschuldung folgt, die aufgrund von Kriegen, jahrzehntelanger Schuldenwirtschaft oder massiven Währungs-

\footnotetext{
${ }^{1}$ Vgl. Staatsbankrott! Bankrotter Staat? Finanzreform und gesellschaftlicher Wandel in Preußen nach 1806, hrsg. vom geheimen Staatsarchiv Preußischer Kulturbesitz, Berlin 2006, S. 19.

${ }^{2}$ Technisch lag 1923 kein Staatsbankrott vor, da die Schulden zurückgezahlt wurden. Es kann aber von einem „verschleierten“ Staatsbankrott gesprochen werden, da die Schulden durch die Hyperinflation entwertet waren. Ein technischer Staatsbankrott lag von 1932 bis 1953 vor, da in dieser Zeit die Altschulden nicht bedient wurden. Vgl. Carmen M. Reinhart, This Time is Different Chartbook: Country Histories on Debt, Default, and Financial Crises, Cambridge/Mass. 2010, S. 50 (NBER Working Paper 15815); hierzu und zum Folgenden vgl. auch Carmen M. Reinhart/Kenneth S. Rogoff, This Time Is Different. Eight Centuries of Financial Folly, Princeton/Oxford 2009.
} 
und Finanzmarktschwierigkeiten entstanden ist. Ab welchem Punkt die Verschuldung zum Bankrott führt, hängt vom Einzelfall ab. Argentinien besaß im Jahr vor seinem letzten Staatsbankrott eine Schuldenquote - verstanden als Anteil der öffentlichen Schulden am Bruttoinlandsprodukt - von gut 64 Prozent $^{3}$. Das ist historisch gesehen sicherlich kein niedriger Wert, aber für eine entwickelte Volkswirtschaft in der heutigen Zeit nicht besorgniserregend hoch. So lag die japanische Schuldenquote 2011 bei über 200 Prozent $^{4}$, ohne dass ein Staatsbankrott drohte. Japan ist jedoch gemessen an seinem wirtschaftlichen Potential eines der reichsten Länder der Welt und hat sich im Unterschied zu Argentinien überwiegend im Inland verschuldet.

Adam Smith, der Begründer der Volkswirtschaftslehre, beschrieb bereits 1776, wann es zu einem Staatsbankrott kommt:

„Haben Staatsschulden eine übermäßige Höhe erreicht, so ist, glaube ich, kaum ein einziges Beispiel vorhanden, dass sie ehrlich und voll bezahlt worden wären. Die Befreiung der Staatseinnahmen ist, wenn sie überhaupt zuwege gebracht wurde, stets durch einen Bankrott erfolgt, manchmal durch einen eingestandenen, stets aber einen tatsächlichen, wenn auch verdeckten. ${ }^{15}$

Ein Staatsbankrott liegt laut der klassischen Studie des Nationalökonomen Alfred Manes vor, „sobald die ursprünglich versprochene Verzinsung und Rückzahlung zum Nachteil der Gläubiger ohne deren Einwilligung geändert wird $^{\text {“6 }}$. Wenn ein Staat einseitig einen Haircut, also einen Schuldenschnitt, vornimmt, die Zinszahlungen reduziert, den Zeitraum der Tilgung verlängert oder den Kapitaldienst vollständig einstellt, ist der Bankrott offenkundig. Bevor es dazu kommt, versuchen die meisten Regierungen einen zweiten, vermeintlich eleganteren Weg zu gehen ${ }^{7}$ : sie drucken Geldscheine oder treffen andere Maßnahmen zur Erhöhung der Geldmenge. Die Absicht ist klar. Die Inflation soll die Schulden entwerten. Der Staat als der mit Abstand größte Gläubiger profitiert am meisten davon. Gerät die Inflation allerdings außer Kontrolle, droht das Geld seine Funktion als Tauschmittel zu verlieren. Es

\footnotetext{
${ }^{3}$ Vgl. Anne Krueger, Crisis Prevention and Resolution: Lessons from Argentina, Cambridge 2002 (www.imf.org/external/np/speeches/2002/071702.htm).

${ }^{4}$ Vgl. Monatsbericht des Bundesministeriums der Finanzen vom Mai 2012, S. 92.

${ }^{5}$ Adam Smith, Der Wohlstand der Nationen, Frankfurt a.M. 2009, S. 1031; zur Bedeutung von Adam Smith vgl. Weitz (Hrsg.), Bedeutende Ökonomen, S. 1-11.

${ }^{6}$ Alfred Manes, Staatsbankrotte. Wirtschaftliche und rechtliche Betrachtungen, Berlin ${ }^{2} 1919$, S. $40 \mathrm{f}$.

${ }^{7}$ Vgl. allgemein Alberto Alesina, The end of large public debts, in: Francesco Giavazzi/Luigi Spaventa (Hrsg.), High Public Debt. The Italian Experience, Cambridge 1989, S.35-89.
} 
kommt dann, wie in Deutschland 1923/24 und 1948, zum Währungsschnitt, der gleichbedeutend mit einem Staatsbankrott ist.

\section{Deutsche Staatsverschuldung im 20. Jahrhundert}

Die deutsche Staatsverschuldung im 20. Jahrhundert gleicht einer Achterbahnfahrt. Die Finanzierung der beiden Weltkriege trieb die Schuldenquoten auf jeweils mehrere hundert Prozent ${ }^{8}$. Dieser astronomischen Verschuldung entledigte sich der deutsche Staat durch Währungsreformen in der Folge zweier gewaltiger Inflationen'. Seit den 1970er Jahren geht es in der Achterbahn der Staatsverschuldung wieder aufwärts. Mittlerweile erreicht Deutschland Schuldenquoten, die es zu Friedenszeiten noch nie zuvor gehabt hat. Selbst nach großen Rüstungsanstrengungen lag die Verschuldung 1913 bei gut 30 Prozent und 1938 bei 50 Prozent der Wirtschaftsleistung, während 2010 - ohne kriegerische Kraftakte - die Marke von 80 Prozent überschritten wurde.

Nur ein einziges Mal in der deutschen Finanzgeschichte des 20. Jahrhunderts wies die Entwicklung der Schuldenquote eine abnehmende Tendenz auf, und zwar in den 1950er Jahren. Die von Fritz Schäffer (CSU), Bundesfinanzminister zwischen 1949 und $1957^{10}$, angehäuften Haushaltsüberschüsse wurden als „Juliusturm“ berühmt - benannt nach der Zitadelle in Berlin-Spandau, wo der aus den französischen Reparationen gespeiste Kriegsschatz des Kaiserreichs gehortet worden war ${ }^{11}$. Wirtschaftswachstum

\footnotetext{
${ }^{8}$ Vgl. Konrad Roesler, Die Finanzpolitik des Deutschen Reiches im Ersten Weltkrieg, Berlin 1967; Manfred Zeidler, Die deutsche Kriegsfinanzierung 1914 bis 1918 und ihre Folgen, in: Wolfgang Michalka (Hrsg.), Der Erste Weltkrieg. Wirkung, Wahrnehmung, Analyse, München/Zürich 1994, S. 415-433; Willi A. Boelcke, Die Kosten von Hitlers Krieg. Kriegsfinanzierung und finanzielles Kriegserbe in Deutschland 1933-1948, Paderborn 1985.

${ }^{9}$ Vgl. Carl-Ludwig Holtfrerich, Bewältigung der deutschen Staatsbankrotte 1918 und 1945, in: Erhard Kantzenbach (Hrsg.), Staatsüberschuldung. Referate gehalten auf dem Symposium der Joachim Jungius-Gesellschaft der Wissenschaften Hamburg am 9. und 10. Februar 1996, Göttingen 1996, S. 27-57.

${ }^{10}$ Vgl. Dieter Grosser, Die Rolle Fritz Schäffers als Finanzminister in den ersten beiden Kabinetten Konrad Adenauers, in: Wolfgang J. Mückl (Hrsg.), Föderalismus und Finanzpolitik. Gedenkschrift für Fritz Schäffer, Paderborn u.a. 1990, S.67-81, sowie Christoph Henzler, Fritz Schäffer, 1945-1967. Eine biographische Studie zum ersten bayerischen Nachkriegs-Ministerpräsidenten und ersten Finanzminister der Bundesrepublik Deutschland, München 1995.

${ }^{11}$ Vgl. Wilhelm Pagels, Der „Juliusturm“. Eine politologische Fallstudie zum Verhältnis von Ökonomie, Politik und Recht in der Bundesrepublik, Diss., Hamburg 1979.
} 
und hohe Steuersätze sicherten die Einnahmen, während der Ausbau des Sozialstaats und der Aufbau der Bundeswehr noch keine großen Summen verschlangen. Doch Schäffers sparsame Haushaltspolitik wurde bald als altmodisch diskreditiert. Bereits sein Nachfolger Franz Etzel (CDU) agierte „hart am Rande des Defizits “'2. Der Verlauf der Schuldenquote wies in den 1960er Jahren aufgrund des hohen Wirtschaftswachstums und der relativ niedrigen Neuverschuldung dennoch nur eine leicht steigende Tendenz auf. Der endgültige Weg in den Schuldenstaat begann in den 1970er Jahren. Die langsamer wachsenden Steuereinnahmen reichten nicht mehr, um die politisch gewünschten Mehrausgaben zu decken. Den Verwerfungen der Weltwirtschaft begegnete man mit immer neuen Konjunkturpaketen, die jedoch keinen nachhaltigen Erfolg brachten. In den 1980er Jahren erfolgte eine Konsolidierung ${ }^{13}$, bevor die Wiedervereinigung die Schuldenquote ab 1990 in ungeahnte Höhen trieb. In nur sechs Jahren, also von 1989 bis 1995, verdoppelte sich die Staatsschuld von 500 Milliarden Euro auf eine Billion Euro ${ }^{14}$. Die stark steigende Schuldenquote der 1990er Jahre war das problematische Ergebnis des hohen Kreditanteils an der Finanzierung der deutschen Einheit ${ }^{15}$.

Erst unter Finanzminister Hans Eichel (SPD) schien die Haushaltskonsolidierung voranzukommen. Das ehrgeizige Ziel eines im Jahr 2006 ausgeglichenen Haushalts, die Durchsetzung eines weitreichenden Sparpakets

${ }^{12}$ Dietrich Yorck, Franz Etzel als Finanzpolitiker, in: Historisch-politische Mitteilungen 2 (1995), S. 173-187, hier S. 181 und S. 183. Vgl. auch Alexander Nützenadel, Stunde der Ökonomen. Wissenschaft, Politik und Expertenkultur in der Bundesrepublik 1949-1974, Göttingen 2005, S. 263 und S. 267.

${ }^{13}$ Vgl. Ulrich Suntum, Finanzpolitik in der Ära Stoltenberg, Bochum 1989, S. 30.

${ }^{14}$ Vgl. Finanzen und Steuern. Fachserie 14, Reihe 5: Schulden der öffentlichen Haushalte 2009, hrsg. vom Statistischen Bundesamt, Wiesbaden 2010, Tabelle 1.1.1. Erläuterung: Schulden einschließlich Kassenkredite.

${ }^{15}$ Die Finanzierung der deutschen Einheit über Kredite wird in der Wissenschaft überwiegend kritisch gesehen. Vgl. z.B. Gerhard A. Ritter, Der Preis der deutschen Einheit. Die Wiedervereinigung und die Krise des Sozialstaats, München 2., erweiterte Aufl. 2007; Reimut Zohlnhöfer, Der lange Schatten der schönen Illusion: Finanzpolitik nach der deutschen Einheit, 1990-1998, in: Leviathan 28 (2000), S. 14-38; Roland Sturm, Die Wende im Stolperschritt. Eine finanzpolitische Bilanz, in: Göttrik Wewer (Hrsg.), Bilanz der Ära Kohl. Christlich-liberale Politik in Deutschland 1982-1998, Opladen 1998, S.183-200; Wolfgang Kitterer, Rechtfertigung und Risiken einer Finanzierung der deutschen Einheit durch Staatsverschuldung, in: Karl-Heinrich Hansmeyer (Hrsg.), Finanzierungsprobleme der deutschen Einheit, Bd. 1: Staatsverschuldung, EG-Regionalfonds, Treuhandanstalt, Berlin 1993, S. 39-76. Dagegen Walther Otremba, Finanzpolitik 1989-1998 - die Dämme haben gehalten, in: Wirtschaftsdienst 79 (1999), S. 18-26. 
Abbildung 1: Entwicklung der Schuldenquote Deutschlands, 1913-2010

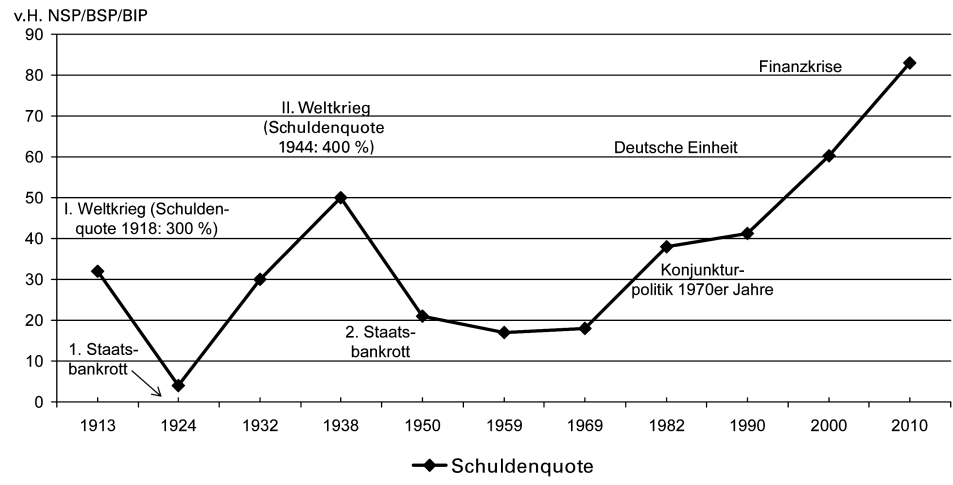

und der überraschend hohe Erlös aus der Versteigerung von neuen Mobilfunklizenzen ließ die Presse wahlweise vom „eisernen Hans“, „Hans Blair“ oder „Hans im Glück“ sprechen ${ }^{17}$. Doch das Glück war - wie so häufig bei Finanzministern - nur flüchtig. Dem Boom der New Economy folgte eine quälend lange Rezession, die zu hohen Haushaltsdefiziten führte ${ }^{18} ; 2006$ wurde die Schulden-Marke von 1,5 Billionen Euro durchbrochen ${ }^{19}$. Statt

${ }^{16}$ Für die Jahre 1913, 1924, 1932, 1938, 1950, 1969 und 1982 vgl. Friedrich-Wilhelm Henning, Staatsfinanzen in historischer Perspektive, in: Klaus-Dirk Henke (Hrsg.), Zur Zukunft der Staatsfinanzierung, Baden-Baden 1999, S. 35-71, hier S. 64; für 1959 vgl. Rolf Caesar, Öffentliche Verschuldung in Deutschland seit der Weltwirtschaftskrise: Wandlungen in Politik und Theorie, in: Dietmar Petzina (Hrsg.), Probleme der Finanzgeschichte des 19. und 20. Jahrhunderts, Berlin 1989, S.9-55, hier S.22; für die Jahre 1990, 2000 und 2010 vgl. Monatsbericht des Bundesministeriums der Finanzen vom Mai 2012, S. 92.

${ }^{17}$ Vgl. Finanzpolitische Leitplanken. Sieben Wegweiser für eine zukunftsfähige, gerechte und europataugliche Finanzpolitik, hrsg. vom Bundesministerium der Finanzen, Berlin 2000. Als Beispiel für das positive Presseecho vgl. Der Spiegel vom 13.11. 2000, S. $168 \mathrm{ff}$. ${ }^{18}$ Vgl. Werner Eichhorst/Klaus F. Zimmermann, Wirtschaftspolitische Bilanz der rotgrünen Bundesregierung, in: APuZ 43/2005, S.11-17; Achim Truger, Rot-grüne Steuerreformen. Finanzpolitik und makroökonomische Performance - was ist schief gelaufen?, in: Eckhard Hein/Arne Heise/Achim Truger (Hrsg.), Finanzpolitik in der Kontroverse, Marburg 2004, S. 169-208; Reimut Zohlnhöfer, Rot-grüne Finanzpolitik zwischen traditioneller Sozialdemokratie und neuer Mitte, in: Christoph Egle/Tobias Ostheim/Reimut Zohlnhöfer (Hrsg.), Das rot-grüne Projekt. Eine Bilanz der Regierung Schröder 1998-2002, Wiesbaden 2003, S. 193-214.

${ }^{19}$ Vgl. Finanzen und Steuern, 14/5, Tabelle 1.1.1. Erläuterung: Schulden einschließlich Kassenkredite. 
eines ausgeglichenen Haushalts erhielt Eichel blaue Briefe aus Brüssel, da die in Maastricht verbindlich vereinbarte Defizithöchstquote von drei Prozent des Bruttoinlandsprodukts mehrfach überschritten wurde. Erst unter Peer Steinbrück (SPD) verbesserte sich die Haushaltslage merklich. Allerdings kam der Bundeshaushalt selbst in den Steuerboomjahren 2007 und 2008 nicht ohne eine Nettoneuverschuldung aus. Die positive Entwicklung der Bundesfinanzen wurde nicht durch Kürzungen auf der Ausgabenseite erzielt $^{20}$, sondern lag vor allem an den stark steigenden Einnahmen infolge der guten Konjunkturentwicklung und der um drei Prozentpunkte erhöhten Mehrwertsteuer. Die im Herbst 2008 einsetzende Wirtschafts- und Bankenkrise beendete diese Entwicklung jäh. Die Schuldenaufnahme im Jahr 2010 war mit 44 Milliarden Euro zwar um 45 Prozent niedriger als geplant, aber „gleichwohl die höchste Neuverschuldung in der Geschichte der Bundesrepublik Deutschland ${ }^{\text {(221 }}$.

Häufig wird übersehen, dass die Kreditmarktschulden nur einen Teil der Verbindlichkeiten der öffentlichen Hand ausmachen. Daher ist im Rahmen der Generationenbilanzierung versucht worden, die gesamten staatlichen Verpflichtungen zu berechnen. Diese implizite Staatsverschuldung, für die eigentlich Rücklagen zu bilden wären, überstieg 2005 in Deutschland, Frankreich und den USA das Bruttoinlandsprodukt um ungefähr das Zweifache und liegt damit deutlich höher als die Verschuldung am Kreditmarkt ${ }^{22}$. Hingegen besaß die Schweiz überhaupt keine implizite Staatsverschuldung, während Großbritannien zwar eine geringe explizite, aber mit 500 Prozent des Bruttoinlandsprodukts eine extrem hohe implizite Staatsverschuldung verzeichnete.

Die mit der steigenden Lebenserwartung einhergehende längere Rentenbezugsdauer wirkt sich generell belastend auf die Generationenbilanz aus. Die Staaten, die die Rentenansprüche bereits reduziert und die Steigerungsraten auf die eine oder andere Weise gekappt haben, vermochten ihre implizite Verschuldung deutlich zu senken. Durch die Alterung der Gesellschaft steigen zudem die Kosten auf dem Gesundheitssektor. Die USA haben auf-

\footnotetext{
${ }^{20}$ Vgl. Maximilian Grasl/Markus König, Von außen getrieben. Die Finanzpolitik der Großen Koalition 2005-2009, in: Christoph Egle/Reimut Zohlnhöfer (Hrsg.), Die zweite Große Koalition. Eine Bilanz der Regierung Merkel 2005-2009, Wiesbaden 2010, S. 205-233, hier S. 229.

${ }^{21}$ Pressemitteilung Nr. 1/2011 des Bundesministeriums der Finanzen.

${ }^{22}$ Vgl. Bernd Raffelhüschen/Stefan Moog/Christoph Müller, Ehrbare Staaten? Die deutsche Generationenbilanz im internationalen Vergleich: Wie gut ist Deutschland auf die demografische Herausforderung vorbereitet?, Berlin 2010, S. 7.
} 
grund ihrer wachsenden und relativ jungen Bevölkerung weniger ein Rentenproblem als vielmehr ein Problem mit ihrem Gesundheitssystem. Die Finanzierung der Gesundheitsausgaben für die working poor ist ein Hauptbelastungsfaktor für die amerikanische Generationenbilanz. Nicht zufällig beschäftigte sich Barack Obama gleich zu Beginn seiner Präsidentschaft vor allem mit dieser Problematik.

\section{Vergleichspunkte: Die USA und Großbritannien}

Die Entwicklung der amerikanischen Staatsverschuldung ist stark geprägt von Kriegen. Das fing an mit dem Unabhängigkeitskrieg gegen Großbritannien, der auch deshalb gewonnen wurde, weil die neu gegründeten Vereinigten Staaten von Amerika in einem erheblichen Umfang Ressourcen auf dem Finanzmarkt zu mobilisieren vermochten; dies war nicht zuletzt ein Verdienst des ersten amerikanischen Finanzministers Alexander Hamil$\operatorname{ton}^{23}$. Die hohe Kreditfähigkeit der USA war auch innenpolitisch von großer Bedeutung, da es den Bürgern, die immerhin gerade gegen englische Steuern und Zölle rebelliert hatten, kaum zuzumuten war, den Krieg ausschließlich über Steuern zu finanzieren. Der Bürgerkrieg und der Erste Weltkrieg schraubten die Schuldenquote auf jeweils fast 40 Prozent. Nach beiden Kriegen wurden die Haushalte ausgeglichen und Altschulden getilgt. Erst in Folge der Weltwirtschaftskrise entstanden größere Defizite. Franklin D. Roosevelts New Deal setzte zwar auf einen Ausbau des Sozialstaats und groß angelegte Arbeitsbeschaffungsmaßnahmen, nicht aber auf keynesianisches Deficit spending ${ }^{24}$. Die Verschuldung explodierte erst, als die USA in den Zweiten Weltkrieg eintraten. Innerhalb von wenigen Jahren verdreifachte sich die Schuldenquote von 40 auf 120 Prozent.

Die amerikanische Entwicklung von 1945 bis 1975 ist ein anschauliches Beispiel dafür, wie ein Staat aus seinen Schulden herauswachsen kann. Der lang anhaltende Wirtschaftsboom, Inflation ${ }^{25}$, eine niedrige Neuverschuldung und mitunter Haushaltsüberschüsse sorgten dafür, dass die Schuldenquote wieder unter 40 Prozent sank. Selbst die von Lyndon B. Johnson eingeführten

\footnotetext{
${ }^{23}$ Vgl. Robert E. Wright, One nation under debt. Hamilton, Jefferson, and the History of What We Owe, New York u.a. 2008, S. 123-160; zum Folgenden vgl. ebenda, S. 42 .

${ }^{24}$ Vgl. Price Fishback, US monetary and fiscal policy in the 1930s, in: Oxford Review of Economic Policy 26 (2010), S. 385-413, hier S. 386 und S. 401-407.

${ }^{25}$ Vgl. Joshua Aizenman/Nancy Marion, Using Inflation to Erode the U.S. Public Debt, Cambridge/Mass. 2009 (NBER Working Paper 15562).
} 
Abbildung 2: Entwicklung der Schuldenquote der USA, 1792-2015

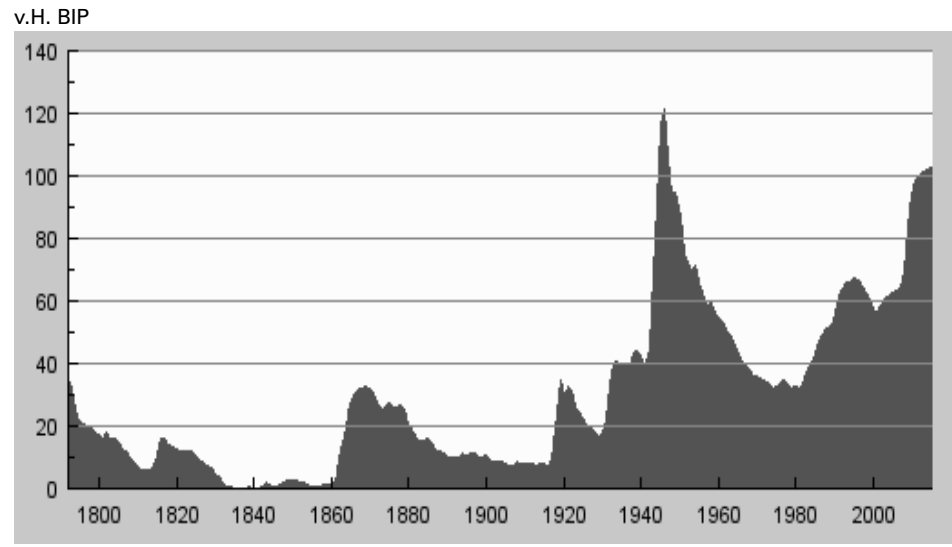

jpgraph

usgovernmentspending.com

Sozialprogramme ${ }^{26}$ und der Vietnamkrieg führten nicht dazu, dass die Schuldenquote deutlich wuchs. Allerdings stieg die Inflation an, was letztlich 1971/ 73 zum Scheitern des Weltwährungssystems von Bretton Woods führte ${ }^{27}$.

Erst der Rüstungskeynesianismus unter Ronald Reagan - also bewusst in Kauf genommene Haushaltsdefizite, die vor allem durch Steuersenkungen und durch den forcierten Rüstungswettlauf mit der Sowjetunion entstanden sind - ließ die Schuldenquote mit Beginn der 1980er Jahre deutlich steigen $^{28}$. Ende der 1990er Jahre wurden das erste Mal wieder Haushaltsüberschüsse erzielt. Bill Clintons Finanzminister Larry Summers rechnete sogar

${ }^{26}$ Vgl. Franz-Xaver Kaufmann, Der deutsche Sozialstaat im internationalen Vergleich, in: Geschichte der Sozialpolitik in Deutschland seit 1945, Bd. 1: Grundlagen der Sozialpolitik, hrsg. vom Bundesministerium für Arbeit und Sozialordnung und dem Bundesarchiv, Baden-Baden 2001, S. 799-989, hier S. 864.

${ }^{27}$ In Bretton Woods wurde 1944 ein festes Wechselkurssystem mit dem auf Gold basierenden Dollar als Leitwährung vereinbart. 1971 gaben die USA die Goldbindung des Dollars auf. 1973 fand das System von Bretton Woods auch formell sein Ende. Die Währungen sind seit 1971/73 nicht mehr in festen Wechselkursen miteinander verbunden, sondern floaten frei. Vgl. Harold James, Rambouillet, 15. November 1975. Die Globalisierung der Wirtschaft, München 1997, S.131ff., sowie Barry Eichengreen, Vom Goldstandard zum Euro. Die Geschichte des internationalen Währungssystems, Berlin 2000, S. 176-182.

${ }^{28}$ Vgl. Uwe Wagschal/Georg Wenzelburger, Haushaltskonsolidierung, Wiesbaden 2008, S. $114 \mathrm{ff}$. und Abbildung 2. 
stolz vor, bis wann sich die USA komplett entschuldet hätten. Die amerikanische Schuldenquote sank zwischen 1993 und 2001 um 17 Prozentpunkte. Für den Demokraten Bill Clinton besaß das Ziel des Haushaltsausgleichs einen höheren Stellenwert als für seine republikanischen Vorgänger und seinen republikanischen Nachfolger.

Die jüngere US-Geschichte ist ein gutes Beispiel, wie schnell sich finanzpolitische Vorzeichen ändern können. Die in absoluten Zahlen riesigen Haushaltsdefizite in der Regierungszeit von George W. Bush führten zunächst zu keiner auffälligen Erhöhung der Schuldenquote, da die amerikanische Wirtschaft stark wuchs. Die Gründe für die Haushaltsdefizite sind vor allem in dem 2001 proklamierten Krieg gegen den Terrorismus und den Steuersenkungen zu suchen. Erst seit der 2007 einsetzenden Rezession und der folgenden Finanzkrise stieg die Schuldenquote beinahe exponentiell. Mit dem Deficit spending wurde eine aktive Konjunkturpolitik finanziert, die verhindern sollte, dass die amerikanische Wirtschaft völlig abstürzte. Zudem verschlang die Stützung der Banken- und Versicherungsbranche sowie wichtiger Unternehmen der sogenannten Realwirtschaft enorm viel Geld.

Die Entwicklung der britischen Schuldenquote wurde vom 17.Jahrhundert bis zur Mitte des 20. Jahrhunderts geprägt vom Kampf gegen Frankreich und Deutschland. Nicht zufällig beginnt die folgende Abbildung zur Entwicklung der britischen Schuldenquote mit dem Jahr 1688. Mit der „Glorreichen Revolution“ wurden die Eigentumsrechte endgültig bestätigt. Das Parlament garantierte seitdem die Staatsschuld und ließ sich fortan nicht mehr bei der Steuerpolitik übergehen ${ }^{29}$.

Wenig später wurde zudem die Bank of England gegründet. Als Ergebnis der "Glorreichen Revolution“ war der englische Staat fast unbegrenzt kreditwürdig $^{30}$, während die Zahlungsmoral der absolutistischen Könige Frankreichs als nicht besonders hoch eingestuft wurde. Zudem erhöhte die frühe Parlamentarisierung die englische Steuermoral und begünstigte den Aufbau einer

\footnotetext{
${ }^{29}$ Vgl. Hans-Christoph Schröder, Die Revolutionen Englands im 17. Jahrhundert, Frankfurt a. M. 1986, S. 233-235.

${ }^{30}$ Vgl. Douglass C. North/Barry R. Weingast, Constitutions and Commitment: The Evolution of Institutions Governing Public Choice in Seventeenth-Century, in: The Journal of Economic History 49 (1989), S. 803-832. Die These von North und Weingast problematisiert Ron Harris, Government and the economy, 1688-1850, in: Roderick Floud/Paul Johnson (Hrsg.), The Cambridge Economic History of Modern Britain, Bd. 1: Industrialisation, 1700-1860, Cambridge ${ }^{4} 2008$, S. 204-237, hier S. 226235.
} 
Abbildung 3: Entwicklung der Schuldenquote Großbritanniens, 1688-2010 31

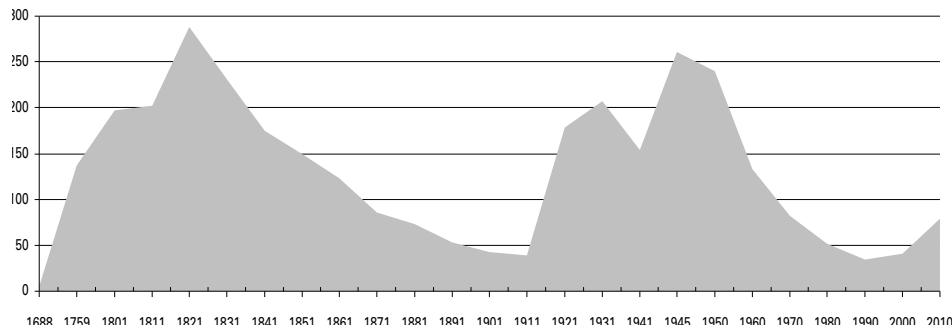

professionellen Steuerverwaltung ${ }^{32}$. Am Ende besiegte Großbritannien Frankreich in den zahlreichen Kriegen bis 1815 und wurde zur unumstrittenen Weltmacht, weil es seine finanziellen Ressourcen weit besser und in einem weit höheren Umfang mobilisieren konnte ${ }^{33}$. Zudem beschleunigte der frühe Aufbau des britischen Finanzmarkts die Industrielle Revolution ${ }^{34}$ und schuf eine Branche, die bis heute von zentraler Bedeutung für die britische Wirtschaft ist.

Die Leistungsfähigkeit des britischen Finanzsystems zeigte sich daran, dass es nach dem Siebenjährigen Krieg, dem amerikanischen Unabhängigkeitskrieg und den napoleonischen Kriegen eine Schuldenquote von fast 300 Prozent zu finanzieren vermochte. Eiserne Haushaltsdisziplin und hohes Wirtschaftswachstum sorgten dafür, dass diese Schuldenquote im 19. Jahrhundert kräftig sank. Auch nach dem Ersten Weltkrieg verschwendete die britische Politik keinen Gedanken daran, die Schulden durch Inflation oder gar einen Währungsschnitt loszuwerden. Im Gegenteil, durch Deflation und Haushaltsausgleich gelang es Schatzkanzler Winston Churchill im Jahr 1925, den Goldstandard wieder zum Vorkriegskurs einzuführen. Der Preis dafür war allerdings hoch: Die Arbeitslosigkeit blieb während der gesamten Zwischenkriegszeit drückend, und die sozialen Spannungen kulminierten im Generalstreik von 1926. Bereits 1931 musste Großbritannien den Gold-

${ }^{31}$ Für die Jahre bis 1990 vgl. Albrecht Ritschl, Sustainability of High Public Debt: What the Historical Record Shows, London 1996, S. 21 (CEPR Discussion Papers 1357); für 2000 und $2010 \mathrm{vgl}$. den Monatsbericht des Bundesministeriums der Finanzen vom Januar 2011, S. 87.

${ }^{32}$ Vgl. John Brewer, The Sinews of Power. War, Money and the English State, 16881783, London 1988.

${ }^{33}$ Vgl. Paul Kennedy, Aufstieg und Fall der großen Mächte. Ökonomischer Wandel und militärischer Konflikt von 1500 bis 2000, Frankfurt a. M. 1989, S. 137-146.

${ }^{34}$ Vgl. North/Weingast, Constitutions and Commitment, S. 831. 
standard wieder aufgeben, was sich auf die wirtschaftliche Entwicklung der 1930er Jahre allerdings positiv auswirkte ${ }^{35}$.

Ähnlich wie im Deutschen Reich stellte sich auch für Großbritannien die Frage, ob ein Deficit spending statt des harten Sparkurses die richtige Antwort auf die Weltwirtschaftskrise gewesen wäre. In Großbritannien gab es zumindest eine politische Alternative, da die Liberalen - beraten und unterstützt von John Maynard Keynes ${ }^{36}$ - mit dem Slogan in den Wahlkampf von 1929 gezogen sind: „We can conquer unemployment ${ }^{\text {“37 }}$. In den 1930er Jahren setzte ein Aufschwung ein, aber wie in den USA und Deutschland sorgte erst die überwiegend kreditfinanzierte Aufrüstung für Vollbeschäftigung $^{38}$.

Die Finanzierung des Zweiten Weltkriegs ließ die Schuldenquote auf über 250 Prozent empor schnellen. Inflation ${ }^{39}$ und günstige weltwirtschaftliche Rahmenbedingungen ermöglichten nach Kriegsende eine relativ schmerzfreie Reduzierung der Schuldenquote, auch wenn die goldenen Jahrzehnte nach dem Zweiten Weltkrieg in Großbritannien weniger ausgeprägt waren als beispielsweise in den USA oder Westdeutschland und das britische Pfund häufiger abgewertet werden musste ${ }^{40}$. Ein keynesianischer

${ }^{35}$ Vgl. allgemein Barry Eichengreen, Golden Fetters. The Gold Standard and the Great Depression, 1919-1939, New York/Oxford 1992.

${ }^{36}$ Vgl. John Maynard Keynes, Can Lloyd George Do It?, in: The Collected Writings of John Maynard Keynes, Bd.9: Essays in Persuasion, Cambridge 1972, S. 86-125. Zur Bedeutung von John Maynard Keynes vgl. Weitz (Hrsg.), Bedeutende Ökonomen, S. 129-140.

${ }^{37}$ Laut Berechnungen von Dimsdale und Horsewood wären bei einem groß angelegten Konjunkturprogramm zwar nicht 500000 zusätzliche Arbeitsplätze entstanden, wie von Keynes vorhergesagt, aber immerhin 330000. Vgl. Nicholas H. Dimsdale/Nicholas Horsewood, Fiscal policy and employment in interwar Britain: some evidence from a new model, in: Oxford Economic Papers 47 (1995), S.369-396. Eher skeptisch: Roger Middleton, Towards the managed economy: Keynes, the Treasury and the fiscal policy debate of the 1930s, London 1985, sowie Roger Middleton, British monetary and fiscal policy in the 1930s, in: Oxford Review of Economic Policy 26 (2010), S.414441 .

${ }^{38}$ Vgl. Barry Eichengreen, The British economy between the wars, in: Floud/Johnson (Hrsg.), Cambridge Economic History of Modern Britain, Bd.1, S.314-343, hier S. 337 .

${ }^{39}$ Vgl. Barry Eichengreen u.a., Public Debts: Nuts, Bolts and Worries, London 2011, S. 62 .

${ }^{40}$ Vgl. Tom Clark/Andrew Dilnot, British fiscal policy since 1939, in: Roderick Floud/ Paul Johnson (Hrsg.), The Cambridge Economic History of Modern Britain, Bd.3: Structural Change und Growth, 1939-2000, Cambridge ${ }^{3} 2009$, S.368-398, hier S.383385 und S. $387 f$. 
Ansatz dominierte die Finanz- und Wirtschaftspolitik der 1950er und 1960er Jahre.

Hohe Inflationsraten, große Schwierigkeiten mit der Währung, steigende Arbeitslosigkeit, ständige Streiks und der Decline der britischen Wirtschaft prägten die Finanzpolitik der 1970er Jahre. Premierminister James Callaghan stellte bereits 1976 auf einem Parteitag seiner Labour Party das Ende der „cosy world [...] where full-employment would be guaranteed by a stroke of the Chancellor's pen“ fest" ${ }^{41}$. Zum endgütigen Kurswechsel kam es 1979 mit der Wahl von Margaret Thatcher ${ }^{42}$. Nicht mehr John Maynard Keynes, sondern Friedrich von Hayek und Milton Friedman waren nun die Ökonomen der Zeit ${ }^{43}$. Die finanz- und wirtschaftspolitische Agenda wurde dominiert von Privatisierung, Deregulierung, Inflationsbekämpfung, Steuersenkungen und Kürzungen der Sozialleistungen. Diese Politik wurde später auch durch Tony Blair im Grunde nicht in Frage gestellt. Allerdings stiegen unter New Labour die öffentlichen Ausgaben; damit wuchs aber auch der staatliche Sektor erheblich, was teilweise über Steuererhöhungen und ab 2000 vor allem über Neuverschuldung finanziert wurde ${ }^{44}$.

Die wirtschaftlichen Rahmenbedingungen waren für die LabourRegierung günstig. Während Blairs Amtszeit wuchs die britische Wirtschaft zumeist deutlich kräftiger als die deutsche und französische. Die gute Konjunkturentwicklung basierte im Wesentlichen auf dem Finanz- und Immobiliensektor. Die Schuldenquote sank auf rund 40 Prozent - ein Wert, der für Schatzkanzler Gordon Brown ,a ,stable and prudent' level“ war und nicht überschritten werden sollte ${ }^{45}$. Die Lage änderte sich jedoch schlagartig mit der Wirtschafts- und Bankenkrise, die in Großbritannien bereits 2007 einsetzte. Allein um den Bankensektor zu retten, musste das Vereinigte Königreich weit mehr öffentliche Gelder einsetzen als seine europäischen Nachbarn ${ }^{46}$. Innerhalb von nur vier Jahren wuchs die britische Schuldenquote von 44,5 auf 77,8 Prozent im Jahr 2010 und überholte damit diejenige Deutschlands ${ }^{47}$.

${ }^{41}$ Zit. nach Clark/Dilnot, British fiscal policy since 1939, S. 390.

${ }^{42}$ Zur britischen Finanzpolitik von 1979 bis 2007 vgl. Reimut Zohlnhöfer, Globalisierung der Wirtschaft und finanzpolitische Anpassungsreaktionen in Westeuropa, Baden-Baden 2009, S. 61-140.

${ }^{43}$ Vgl. Weitz (Hrsg.), Bedeutende Ökonomen, S. 141-148 und S. 169-178.

${ }^{44}$ Vgl. Zohlnhöfer, Globalisierung der Wirtschaft, S. 119-125.

${ }^{45}$ Zit. nach Clark/Dilnot, British fiscal policy since 1939, S. 394.

${ }^{46}$ Vgl. Yvonne Esterházy, Großbritannien und die Folgen der Finanzkrise, in: APuZ 49/2010, S. 40-46.

${ }^{47}$ Vgl. Monatsbericht des Bundesministeriums der Finanzen vom Januar 2011, S. 87. 
Die USA und Großbritannien haben im Unterschied zu Deutschland nie einen Staatsbankrott erklärt und nie ihre Währung riskiert. Aus den hohen, vor allem aus Kriegen stammenden Schulden wuchsen beide Staaten heraus. Eine lange Zeit auf Haushaltsausgleich orientierte Finanzpolitik unterstütze diesen Prozess. 
